\title{
Episodic medical home interventions in severe bedridden Chronic Respiratory Failure patients: a 4 year retrospective study
}

\author{
L. Barbano, E. Bertella, M. Vitacca
}

ABSTRACT: Episodic medical home interventions in severe bedridden Chronic Respiratory Failure patients: a 4 year retrospective study. L. Barbano, E. Bertella, M. Vitacca.

Background and Aim. Home care for respiratory patients includes a complex array of services delivered in an uncontrolled setting. The role of a respiratory specialist inside the home healthcare team has been scarcely studied up to now. Our aims were to analyse the number and quality of episodic home visits performed by respiratory physicians to severe bedridden Chronic Respiratory Failure (CRF) patients, and also to evaluate the safety of tracheotomy tube substitutions at home.

Methods. 231 home interventions (59.8/year) in 123 CRF patients (59 males; age $63 \pm 17 y, 24$ on oxygen therapy, 35 under non invasive mechanical ventilation, 46 under invasive ventilation, 74 with tracheostomy) located $35 \pm 16 \mathbf{~ k m}$ far from referred hospital, were revised in a period of 4 years $(2005-2008)$.

Results. Chronic Obstructive Pulmonary Disease (COPD) $(31 \%)$ and amyotrophic lateral sclerosis (ALS) $(\mathbf{2 8 \%})$ were the more frequent diagnoses. Interventions were: tracheotomy tube substitution (64\%) presenting $22 \%$ of minor adverse events and $1.4 \%$ of major adverse events; change or new oxygen prescription (37\%); nocturnal pulsed saturimetric trend prescription $(24 \%)$; change in mechanical ventilation (MV) setting (4\%); new MV adaptation $(7 \%)$. After medical intervention, new home medical equipment devices (oxygen and MV) were prescribed in $36 \%$ of the cases while rehabilitative hospital admission and home respiratory physiotherapy prescription was proposed in $9 \%$ and $6 \%$ of the cases respectively. Patient/caregiver's satisfaction was reported on average $8.48 \pm 0.79(1=$ the worst; $10=$ the higher $)$. The local health care system (HCS) reimbursed 70€ for each home intervention. Families saved $42 \pm 20 €$ per visit for ambulance transportation.

Conclusions. Home visits performed by a respiratory physician to bedridden patients with chronic respiratory failure:

1. include predominantly patients affected by COPD and ALS;

2. determine a very good satisfaction to patients/caregivers;

3. allow money saving to caregivers;

4. are predominantly made up to change tracheotomy tube without severe adverse events.

Monaldi Arch Chest Dis 2009; 71: 3, 113-118.

Key words: Home care, Respiratory Failure, Home visits.

Respiratory Unit and Weaning Center, Fondazione Salvatore Maugeri, IRCCS, Lumezzane (BS), Italy.

Correspondence: Dr. Michele Vitacca, Respiratory Unit and Weaning Centre, Fondazione Salvatore Maugeri, Via Mazzini 129, 25066 Lumezzane (BS), Italy; e-mail:michele.vitacca@fsm.it.

\section{Introduction}

Disabled older adults continue to grow and over the next 20 years we will have to manage chronic disease in an ageing population. This can heavily affect the economic resources of the health system. To offer continuity of care it is important to improve home management of these frail and chronically ill patients. Respiratory patients represent an heavy burden to their caregivers for their dependency and the complexity of care they need due to mechanical ventilation (MV), presence of tracheotomy tube, acute exacerbations, distance to hospital [1-2]. Respiratory patients at home require a complex array of care to be provided in an uncontrolled setting. In this setting patients are the centre of the home care program, but sometimes patients and families can be integral member of their own management [3].

A recent ATS statement [3] has underlined the necessity to provide homecare focusing on a pa- tient-centred perspective and patient and family satisfaction. It indicates as major end points reduction of complications resulting from hospital admission, maintaining an acceptable quality of life, and enabling a comfortable and dignified death [3]. Home care is the provision of services and equipment in the place where individuals with needs resulting from acute or chronic illness, permanent disability, or terminal illness and their families live [3]. Home care include episodic, often post-acute, home health care given on an intermittent basis, palliative and end-of-life care, chronic home care services provided on an hourly basis, and home medical equipment prescription [3].

In the USA this service is provided by Medicarecertified home health agencies; nurses or other home health personnel, in particular respiratory, physical or occupational therapists provide skilled care according to federal regulations. Faroux et al [4] reported that in Europe important differences exist between different countries for home treatment of pa- 
tients with chronic respiratory failure. New models of supported discharge from hospital at home programs are being implemented: patients are included in an early hospital or Emergency Room (ER) discharge with follow-up by respiratory specialist nurses through frequent telephone contacts, home visits or telemedicine when patient's condition or ability to manage the care makes it necessary [5-9]. To date in Italy there is a lack of uniformity in national policy for home care programs and the type of care provided [10-12]: home supervision is performed only in some part of the country by nurses and physicians with a local HCS plan [11-12].

Patients have a variety of different needs so they should be addressed using a patient - or family-centered model because home care is provided in the patient's context and the provider is a visitor [3]. Home healthcare providers must assist patients and their caregivers to manage their daily life, recognise and respond to complications and be able to use respiratory equipment safely.

It has been shown that activation of home care programs for chronically ill patients, improves survival [12] quality of life, reduces ER access and reduces the costs of conventional care [8, 12-16], even if few studies denied it for COPD patients $[12,17]$. Until now studies have focused on the effectiveness of home care provided by nurses, even if specialised, but few data exists on visits by specialised physician and in particular on the feasibility and safety of medical intervention at home. In the USA the role of the primary care physician who provides home care has been defined into three main activities:

1. making home visits;

2. referring patients to home care agencies;

3. participation in the multidisciplinary team providing home care.

Primary care physicians make regular visit, but infrequent home visits, specialists may also make home visits to supervise complex care like mechanical ventilation [3].

The present study aims to analyse retrospectively episodic medical home visits to patients with severe chronic respiratory failure who cannot reach the clinic to verify number, quality and safety of interventions performed.

\section{Materials and methods}

\section{Patients}

In the district of the city of Brescia we are considering a program of "structured homecare" has been activated for patients which satisfy the following inclusion criteria:

1. frail patients with frequent hospital admissions;

2. bedridden;

3. with comorbidities;

4. with recent hospital discharge with continuum care;

5. with intensive nursing assistance;

6. with poor family assistance for home mechanical ventilation, tracheotomy tube care and/or long term oxygen therapy (LTOT).
We considered all the patients who received respiratory home visits/interventions by a physician specialist in respiratory medicine employed by "Fondazione S. Maugeri Lumezzane Medical centre (BS)" from January 1 st 2005 to December 31 st 2008.

In the Brescia district 3967 patients were included in a "homecare program" during the 4 years considered by the study: 123 patients $(3.1 \%, 59$ males) aged $63 \pm 17$ (mean \pm SD) received 231 (59.8/year) home visits by 2 physicians specialist in respiratory medicine. All the patients were affected by chronic respiratory failure and the largest part of them had a tracheotomy tube $(60 \%)$. The majority of the patients had COPD $(35 \%)$ and ALS $(28 \%)$, only $2.5 \%$ of the patients had other neuromuscular diseases, while $34 \%$ of the patients had different diagnosis as neurological diseases (tetraplegia, stroke), surgical complications, apallic coma, advanced stage cancer or were geriatric patients presenting several co-morbidities.

\section{Description of the Hospital Unit and the respiratory physicians}

The specialists in respiratory medicine are employed by the Pulmonary Rehabilitation Unit of Fondazione S. Maugeri (Lumezzane) which is made up by a ward with 34 beds for ordinary inpatients, 4 beds of respiratory intensive care unit, a day hospital service, a respiratory clinic for out-patients and a gym for pulmonary rehabilitation activity with 4 respiratory therapists. The main activities of the hospital are pulmonary rehabilitation and weaning from mechanical ventilation. The physicians are expert in invasive and non-invasive mechanical ventilation and in the management of tracheotomy tubes. Patients who refer to the centre are affected by respiratory failure from pulmonary diseases as chronic obstructive pulmonary disease (COPD) and fibrosis and neurological diseases as amyotrophic lateral sclerosis (ALS) and Duchenne Muscular Dystrophy (DMD). If home mechanical ventilation is needed, the physicians follow both the adaptation and the education of patients and care givers.

\section{Description of home visits}

Home visits were requested from the Home HCS district of Brescia to Hospital administrative office. The requests were then given to respiratory specialists. The visits were for no urgent respiratory problems which the general practitioner (GP) could not manage and they were done within 3 days. The candidates for home visits were strictly proposed by GP or by home care staff of the HCS according to the following criteria: quadriplegic patients, severe bedridden patients with difficulties to perform an outpatient visit, presence at home of frail instrumentation as invasive mechanical ventilation, nutritional pumps and suction devices not easily available on ambulance or in an outpatient clinic. The HCS paid 70 Euro for each visit whatever the reason was. 


\section{Description of services (visits and the equipment)}

The physician drove to the location at the allocated time. In selected cases a nurse employed by Home Sanitary Service was there to give assistance. The equipment was made up by a pulsoximeter (Nonin Onyx 9500, Nonin Medical Inc. Plymouth MN USA) and in the case of tracheotomy tube substitution by:

- Tracheotomy tube of the model and size owned by the patient

- Tracheotomy tube of just lower size

- Suction catheters

- Extractor fan (suction device)

- Ambu balloon.

During the visit different therapeutic decision or intervention could be performed:

\section{Tracheotomy tube substitution: a pulsoxime-} ter was used to monitor the oxygen saturation of the patient, an accurate toilette of bronchial secretion was made through the tracheotomy tube and, if necessary the tracheotomy cuff was deflated and the mechanical ventilation suspended. A catheter was used as a guide to extract the cannula and insert the new one. The oxygen saturation was monitored by a pulsoxymeter during the whole intervention. The physician was assisted by a nurse of the home care service or by a previously trained caregiver.

2. $\mathrm{O}_{2}$ prescription modification according to blood gas analysis (in very selected cases) or simply to pulsoximetry data (in case of confirmation of the prescription).

3. $\mathrm{O}_{2}$ prescription suspension as described for prescription modification.

4. Drug therapy modification.

5. Prescription of night oxygen saturation monitoring by trend pulsoxymeter (Nonin Onyx 9500).

6. Mechanical ventilation resetting according to patient's comfort.

7. Adaptation to home non invasive mechanical ventilation for patients with acceptable respiratory autonomy who refused hospital admission; this included a continuous pulsoxymetry control.

8. Prescription of new equipment and devices for MV.

9. Prescription of hospital admission for rehabilitation.

10. Prescription of home respiratory rehabilitation course.

For each visit, doctors wrote a detailed report of their consultancy with details of any interventions carried out, the prescriptions and the possible adverse events. This report was sent to the HCS home care that had required the visit and to patient's GP. At the end of the visit the patient or the caregiver was asked to indicate on a simple nonvalidated graduated scale ranging from 1 to 10 (1 being minimum satisfaction and 10 the maximum) how satisfied they were with the home service.

The entire cost of home-care equipments (mechanical ventilation, suction devices, nutritional pumps, equipments) has been supported by HCS.

\section{Data collection}

Anthropometrical data, diagnosis according to clinical and functional reports (hospital or GP clinical chart), measure or intervention undertaken and the possible side effects/events were collected into a registry of the Pulmonary Rehabilitation Unit. Side effects were defined as "immediate side effect" i.e. clinical problems immediately recorded during the visit or "short term side effect" i.e recorded within $24 \mathrm{H}$. The theoretical money saving for the patient's family has been calculated considering $1 € / \mathrm{km}$ the cost of the ambulance to carry the patient to the clinic according to the current price list used by Emergency Ambulance Association.

All the data was descriptively analysed. Data was expressed as mean \pm SD or percentage of frequency.

\section{Results}

The visits were carried out on an area extended on a radius of $35 \pm 16 \mathrm{~km}$ from our hospital.

Figure 1 shows the time course of number of visits according to the different diagnosis. As shown in figure 1 the number of visits increased in the second year of activity $(+25 \%)$ remaining equally distributed in the last 3 years. While COPD patients were equally distributed among years with a decrease in the last year, the increasing trend of visits for ALS patients $(+183 \%$ in the 2 nd year and plus $29 \%$ in the 3 rd year) stabilised the last 2 years. Patients with miscellaneous diagnosis were present in all years of activity with a decreasing trend from 1 st to others years. The major number of intervention was tracheotomy tube substitution $(64 \%)$. In the $37 \%$ of the cases oxygen prescription has been modified to adjust the oxygen $\left(\mathrm{O}_{2}\right)$ flow according to the actual need of the patient and in some case this therapy has been stopped. In the $36 \%$ of the cases, after accurate evaluation of patient's clinical condition, oxygen or home mechanical ventilation has been prescribed. In the $24 \%$ of the cases a night oxygen saturation monitoring has been suggested to adjust oxygen flow or mechanical ventilation settings when nocturnal hypoventilation was suspected. In the $9 \%$ of the visits it has been necessary to admit the patient to the hospital because of worsening of clinical condition and the impossibility to properly carry on with home care. In the $7 \%$ of the cases the patient has been adapted to non-invasive mechanical ventilation, in the $6 \%$ a home rehabilitation course has been prescribed and in the $4 \%$ of the cases mechanical ventilation settings have been modified according to patient's needs. During the 136 tracheotomy tube substitutions, 30 (22\% of the cases) minor adverse event occurred ( 5 bleeding, 15 transit difficulty because of reduction of the stoma diameter and in 10 cases both events). Only $2(1.4 \%)$ major adverse events occurred: in one case a difficult tracheotomy tube substitution with huge bleeding, made necessary an urgent hospital admission because of the impossibility to 


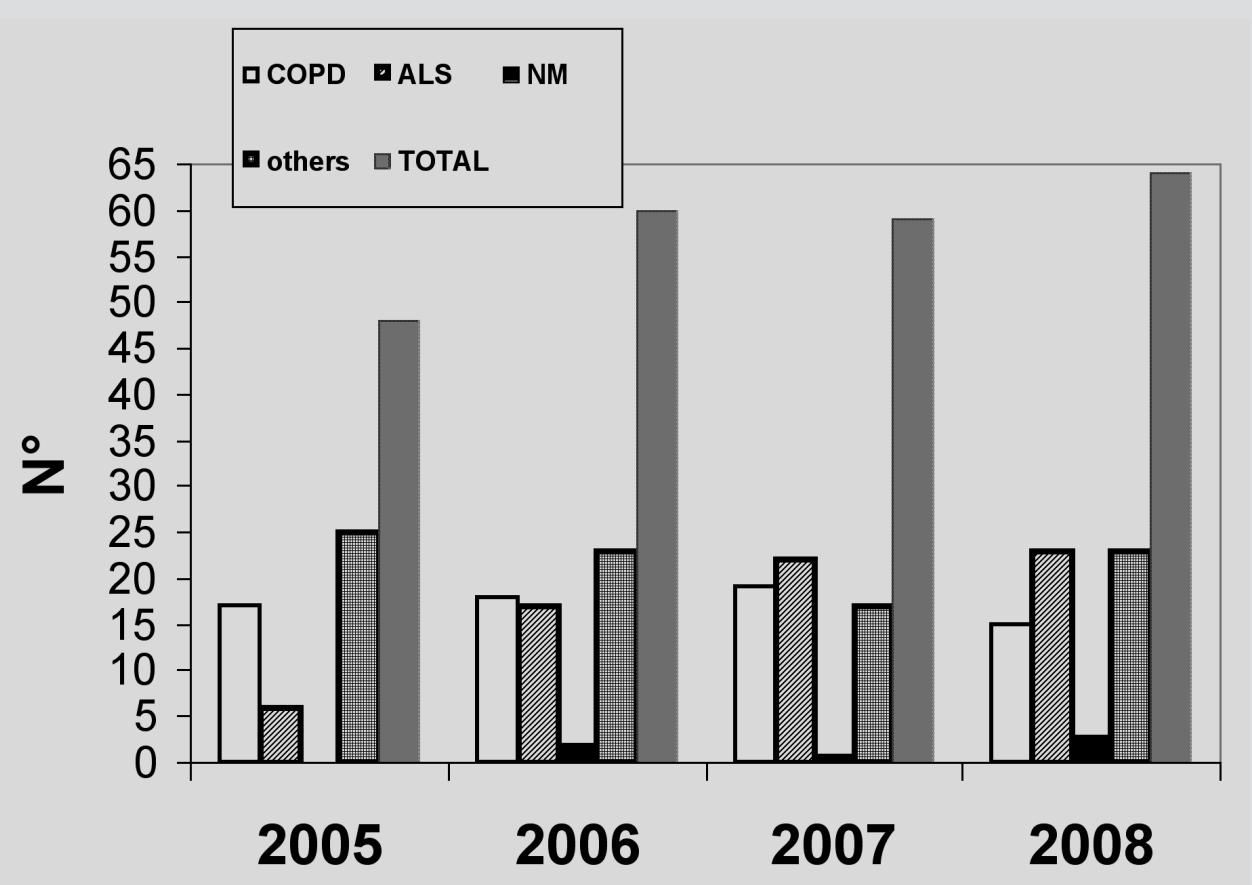

\begin{tabular}{lcccc}
\hline years & 2005 & 2006 & 2007 & 2008 \\
\hline COPD (\%) & $36 \%$ & $31 \%$ & $32 \%$ & $23 \%$ \\
\hline ALS (\%) & $12 \%$ & $28 \%$ & $37 \%$ & $36 \%$ \\
\hline NM (\%) & 0 & $3 \%$ & $2 \%$ & $5 \%$ \\
\hline Others (\%) & $52 \%$ & $38 \%$ & $29 \%$ & $36 \%$ \\
\hline
\end{tabular}

Fig. 1 - Time course of number of visits according to diagnosis (white bars: COPD; line bars: ALS; black: NM; square: others; grey bars: total patients) and among years.

manage the situation at home, in the other case after a fruitless attempt to substitute the tracheotomy tube it has been necessary to put the previous tracheotomy tube again and assist patient ventilation with ambu balloon and the mechanical ventilator in use for temporary acute respiratory failure. The miscellaneous patients presented the highest percentage of side effects (41\%) while the ALS the lowest $(9.8 \%)$; COPD group presented side effects in the $12 \%$ of the cases while NM in the $33 \%$ of the cases. No short term side effects as hypercapnic episode, immediate hospitalization and sudden relapse have been described by relatives, home nurses or GP within 24 hours after the home visit.

The satisfaction for the service was very high as shown by a mean score of $8.48 \pm 0.79$. Only during the $7.3 \%$ of the visits patients expressed a judgment of partial satisfaction giving a score less than 8. The local HCS reimbursed $70 €$ for each home intervention. The interventions/visits performed at home may have been provided in outpatient or inpatient settings with a range of HCS costs/reimbursement from $16 € /$ intervention to $289 € /$ day of reimbursement. Families saved on average $42 \pm 20 €$ (range 15-110€) per visit for ambulance transportation.

\section{Discussion}

Our data provides some interesting information about the candidates who require home visits, the level of quality and safety of these visits and the money saved for patients and caregivers.

When compared to other available literature $[12,13,15,17,19]$ the severity of our patients visited at home was particularly high as demonstrated by the oxygen, mechanical ventilation and tracheostomy necessities.

The majority of patients who received home visits were affected by COPD, but the number of these patients remained constant during the 4 year study, while the number of those with ALS increased greatly becoming the larger group in 2007 and 2008, this trend is probably due to a better knowledge of the disease and thus earlier diagnosis, better assistance to patients and an extension of life expectation. Important data that can be taken from this study is that performing a tracheostomy at home, as opposed to the usual hospital setting, is safe and well tolerated by patients. This is demonstrated by the small number of adverse events both major (two) and minor (thirty) which occurred during the intervention with only one case of ur- 
gent hospital admission. Nevertheless all the visits and the interventions considered were performed by physicians not only specialist in respiratory medicine, but also expert in management of critical patients with respiratory failure and dependent on mechanical ventilation. In fact the described home visits do not only mean simple consultancy, but also interventions that require an expert specialist because of the presence of certain risks during the procedure. Despite the absence of data in support, we believe that the outcome of such interventions is strongly related to the capability and the experience of the operator. This would probably affect the satisfaction levels of patients and caregivers, that in this study appear to be very high being on average 8.48 on a scale from 1 to 10 . Hughes and colleagues have [18] already reported good patient and caregiver outcomes promoted by a hospital based homecare model and we agree with them that this good perception of the service can be achieved with continuity of care and the availability of a specialist to assist the families.

Moreover every home visit described in the present study allowed patients and their families to save on average 42 euro. This money saving was predominantly due to the cost of ambulance transportation being that the patients are often bedridden or dependent on ventilation, thus difficult to transport in their own vehicles. Evaluating the cost saving for HCS was not the aim of the present study; nevertheless we can speculate according to a previous study [12] a money saving both for reduction of hospital admissions, outpatients visits and the possibility of periodically reviewing patient testing according to his therapeutic needs.

Indeed it has been previously shown $[12,19]$ in COPD patients that shifting the place where care are provided from the hospital to the home allows greater cost-efficiency by reducing mortality [12], hospital re-admission [12, 19] and numbers of patients on long-term medication [19]. Such rationalisation of health costs is essential, for example, in the case of changing oxygen or other mechanical ventilation needs. In fact, in some cases the oxygen flow has been reduced or the prescription of oxygen or mechanical ventilation has been suspended. This is not due to incorrect prescriptions, but the variation of the patients' needs also relate to a better awareness of the disease and a better compliance to therapy. The success can also be attributed to home assistance not only by physician but also by nurses and especially by physiotherapists [12]. All this can be due to care quality and to assistance continuity offered by means of co-operation between the people operating at home and people working in hospital [12]. In this way a relationship is created that assures complete and competent assistance to the patient. Up to now various studies have shown the efficacy of homecare to reduce mortality [12] urgent hospital access, improve the quality of life and reduce costs for the health system [13, 15, 16]. Nevertheless these studies focused on nurses, even specialist physiotherapists, but only one study [12] has presented visits by physicians and focused on inter- ventions which require the competence of a specialist. Considering the transport difficulty of patients with chronic respiratory failure, in particular if ventilated or with neuromuscular disease, we hypothesised that home visits by physicians specialist in respiratory medicine besides the nurses assistance, could be an important support for patients and caregivers. Due to the complexity of the clinical problems, we believe that the presence of expert physicians would be essential to offer competent support to the caregivers and the GP and intervene when necessary. Homecare targets for respiratory patients are listed in the 2005 American Thoracic Society document [3]. They include reducing mortality and morbidity, improving functioning and quality of life and education. Moreover home care often fulfills the target to reduce health costs by reducing hospital admission days [20]. It has already been demonstrated that all these goals are reached with the assistance of homecare nurses. Thus our data suggests that in severe respiratory patients, medical visits or interventions at home determine money saving with very good satisfaction. This kind of assistance is performed by a small number of centres and physicians who cannot travel far, so a relatively small area is involved. This is also a limit of our study considering that only one centre collects data relating to a limited area (average distance for a visit $35 \pm 16 \mathrm{Km}$ ). In this way a co-operation system between home, general practitioners and hospital may be developed with complete, competent and prompt assistance reducing, at the same time, unnecessary costs to the health system. As limitation of our study we have proposed a description of a home-care service without control group (i.e. of patients who have been evaluated and treated in hospital) to compare the home-care group for costs, quality of life, patient/caregiver satisfaction, and adverse events.

In conclusion, home visits performed by a respiratory physician to bedridden patients with chronic respiratory failure:

1. include predominantly patients affected by COPD and ALS;

2 determine a very good satisfaction to patients;

3. allow money saving to caregivers;

4. are predominantly made up to change tracheotomy tube without adverse events.

\section{References}

1. Vitacca M, Escarrabill J, Galavotti G, et al. Home mechanical ventilation (HMV) patients: a retrospective survey to identify level of burden in real life. Monaldi Arch Chest Dis 2007; 67: 3, 142-147.

2. Langa KM, Fendrick AM, Flaherty KR. Informal Caregiving for Chronic Lung Disease Among Older Americans. Chest 2002; 122: 2197-2203.

3. ATS Documents: statement on Home Care for Patients with Respiratory Disorders. Am J Respir Crit Care Med 2005; 171, 1443-1464.

4. Faroux B, Howard P, Muir JF. Home treatement for chronic respiratory insufficiency: the situation in Europe in 1992. Eur Resp J 1994; 7: 1721-1726. 
5. Davies L, Wilkinson M, Bonner S, Calverley PMA, Angus RM. "Hospital at home" versus hospital care in patients with exacerbations of chronic obstructive pulmonary disease: prospective randomised controlled trial. BMJ 2000; 321: 1265-1268.

6. Richards SH, Coast J, Gunnell DJ, Peters TJ, Pounsford J, Darlow M-A. Randomised controlled trial comparing effectiveness and acceptability of an early discharge, hospital at home scheme with hospital care. BMJ 1998; 316: 1796-1801.

7. Shepperd S, Harvood D, Jenkinson C, Gray A, Vessey M, Morgan P. Randomised controlled trial comparing hospital at home care with inpatient hospital care. Three month follow-up of health outcomes. BMJ 1998; 316 : 1786-1791.

8. Sala E, Alegre L, Carrera M, et al. Supported discharge shortens hospital stay in patients hospitalized because of an exacerbtaion of COPD. Eur Respir J 2001; 17: $1138-1142$

9. Vitacca M, Bianchi L, Guerra A, et al. Tele-assistance in Chronic Respiratory Failure Patients: a Randomised Clinical Trial. Eur Respir J 2009; 33: 411-418.

10. Donner CF, Pesce L, Zaccaria S, Erbetta M, Mazzetti D. Organisation of home respiratory care in Italy. Monaldi Arch Chest Dis 1993; 48: 5, 468-472.

11. Sturani C. Respiratory home care: reality and perspectives. Rassegna di Patologia dell Apparato Respiratorio 2006; 21: 272-279.

12. Rizzi M, Grassi M, Pecis M, et al. A specific home acre program improves the survival of patients with chronic obstructive pulmonary disease receiving long term oxygen therapy. Arch Phys Med Rehabil 2009; 90: 395401.

13. Hernandez C, Casas A, Escarrabill J, et al. Home hospitalisation of exacerbated chronic obstructive pulmonary disease patients. Eur Respir J 2003; 21: 58-67.

14. Brumley RD, Enguidanos S, Cherin DA. Effectiveness of a home-based palliative care program for end-of-life. J Palliat Med 2003; 6: 715.

15. Farrero E, Escarrabil J, Prats E, Maderal M, Manresa F. Impact of hospital-based home-care program on the management of COPD patients receiving long-term oxygen therapy. Chest 2001; 119: 364-369.

16. Adams SG, Smith PK, Allan PF, Anzueto A, Pugh JA, Cornell JE. Systematic review of the chronic care model in chronic obstructive pulmonary disease prevention and management. Arch Intern Med 2007; 167: 551561.

17. Pearson S, Inglis SC, McLennan SN, et al. Prolonged effects of a home-based intervention in patients with chronic illness. Arch Intern Med 2006; 166: 645-650.

18. Huges SL, Weaver FM, Giobbie-Hurder A, et al. Effectivenes of team-managed home-based primary care. JAMA 2000; 284: 2877-2885.

19. Gordois A, Scuffham P, Gibbons D. The cost-effectiveness of outreach respiratory care for COPD patients. Prof Nurse 2002; 17: 504-507.

20. Huges SL, Ulasevich A, Weaver FM, et al. Impact of home care on hospital days: a meta analysis. Health Serv Res 1997; 324: 415-432.

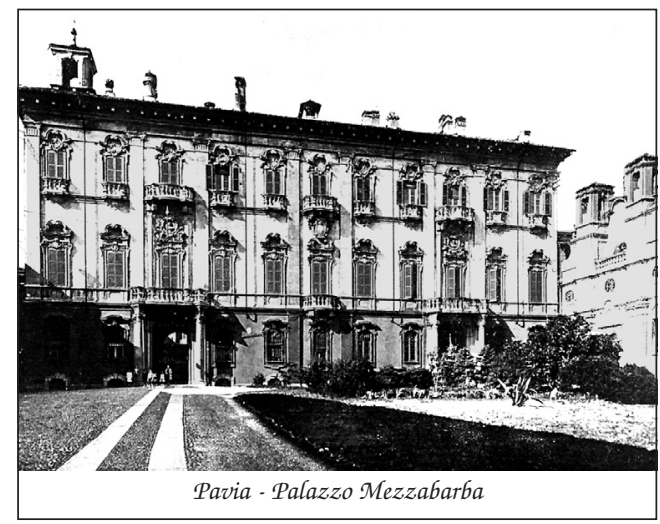

